Manuscript: JO061909o

\title{
Imidazolium ionic liquids as solvents for cerium(IV)-mediated oxidation reactions
}

\author{
Hasan Mehdi, Andrea Bodor, Diána Lantos, István T. Horváth, Dirk E. De Vos, \\ and Koen Binnemans*
}

\section{Supporting information}

\section{CONTENTS}

Page S2: ${ }^{1} \mathrm{H}-\mathrm{NMR}$ spectrum of 1-methylimidazolium hydrogensulfate, $[\mathrm{Hmim}]\left[\mathrm{HSO}_{4}\right]$. Page S3. ${ }^{13} \mathrm{C}-\mathrm{NMR}$ spectrum of 1-methylimidazolium hydrogensulfate, [Hmim] $\left[\mathrm{HSO}_{4}\right]$. Page S4. ${ }^{1} \mathrm{H}-\mathrm{NMR}$ spectrum of 1-butylimidazolium trifluoromethanesulfonate, [Hbim][TfO]. Page S5. ${ }^{13}$ C-NMR spectrum of 1-butylimidazolium trifluoromethanesulfonate, [Hbim][TfO]. Page S6. ${ }^{1} \mathrm{H}-\mathrm{NMR}$ spectrum of 1-hexyl-3-methylimidazolium trifluoromethanesulfonate, $\left[\mathrm{C}_{6} \mathrm{mim}\right][\mathrm{TfO}]$.

Page S7. ${ }^{13} \mathrm{C}-\mathrm{NMR}$ spectrum of 1-hexyl-3-methylimidazolium trifluoromethanesulfonate, $\left[\mathrm{C}_{6} \mathrm{mim}\right][\mathrm{TfO}]$.

Page S8. ${ }^{1}$ H-NMR spectrum of 1-methylimidazolium trifluoromethanesulfonate, [emim][TfO] in acetone- $\mathrm{d}_{6}$. Page S9. ${ }^{13}$ C-NMR spectrum of 1-methylimidazolium trifluoromethanesulfonate, [emim][TfO] in acetone- $\mathrm{d}_{6}$.

Page S10. ${ }^{19}$ F-NMR spectrum of 1-methylimidazolium trifluoromethanesulfonate, [emim][TfO] in acetone- $\mathrm{d}_{6}$. 
Figure S1. ${ }^{1} \mathrm{H}-\mathrm{NMR}$ spectrum of 1 -methylimidazolium hydrogensulfate, $\left[\mathrm{Hmim}^{-}\left[\mathrm{HSO}_{4}\right]\right.$ in dmso-d $_{6}(300 \mathrm{MHz})$.

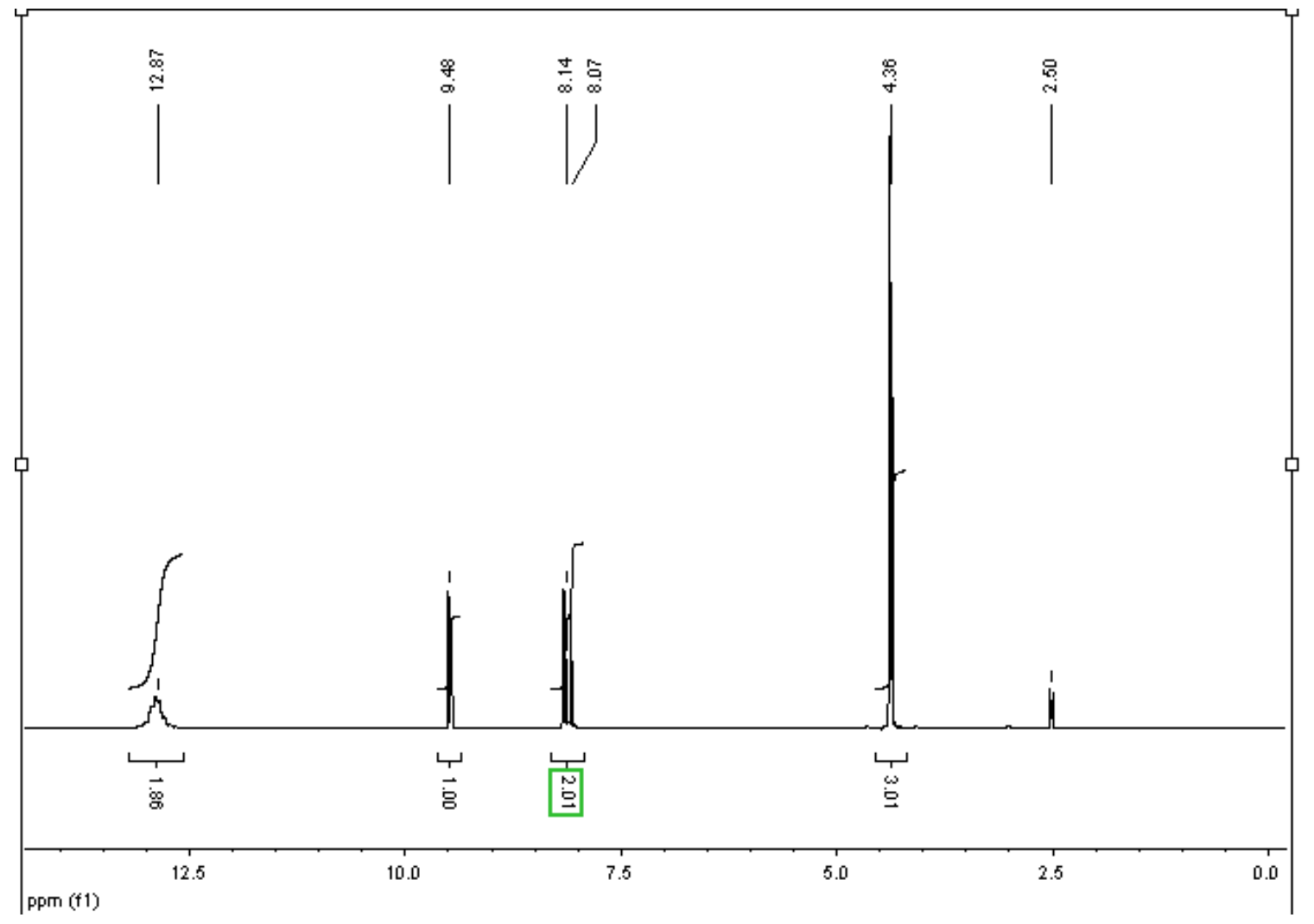


Figure $\mathrm{S} 2 .{ }^{13} \mathrm{C}$-NMR spectrum of 1-methylimidazolium hydrogensulfate, $[\mathrm{Hmim}]\left[\mathrm{HSO}_{4}\right]$ in dmso-d $_{6}(75.47 \mathrm{MHz})$.

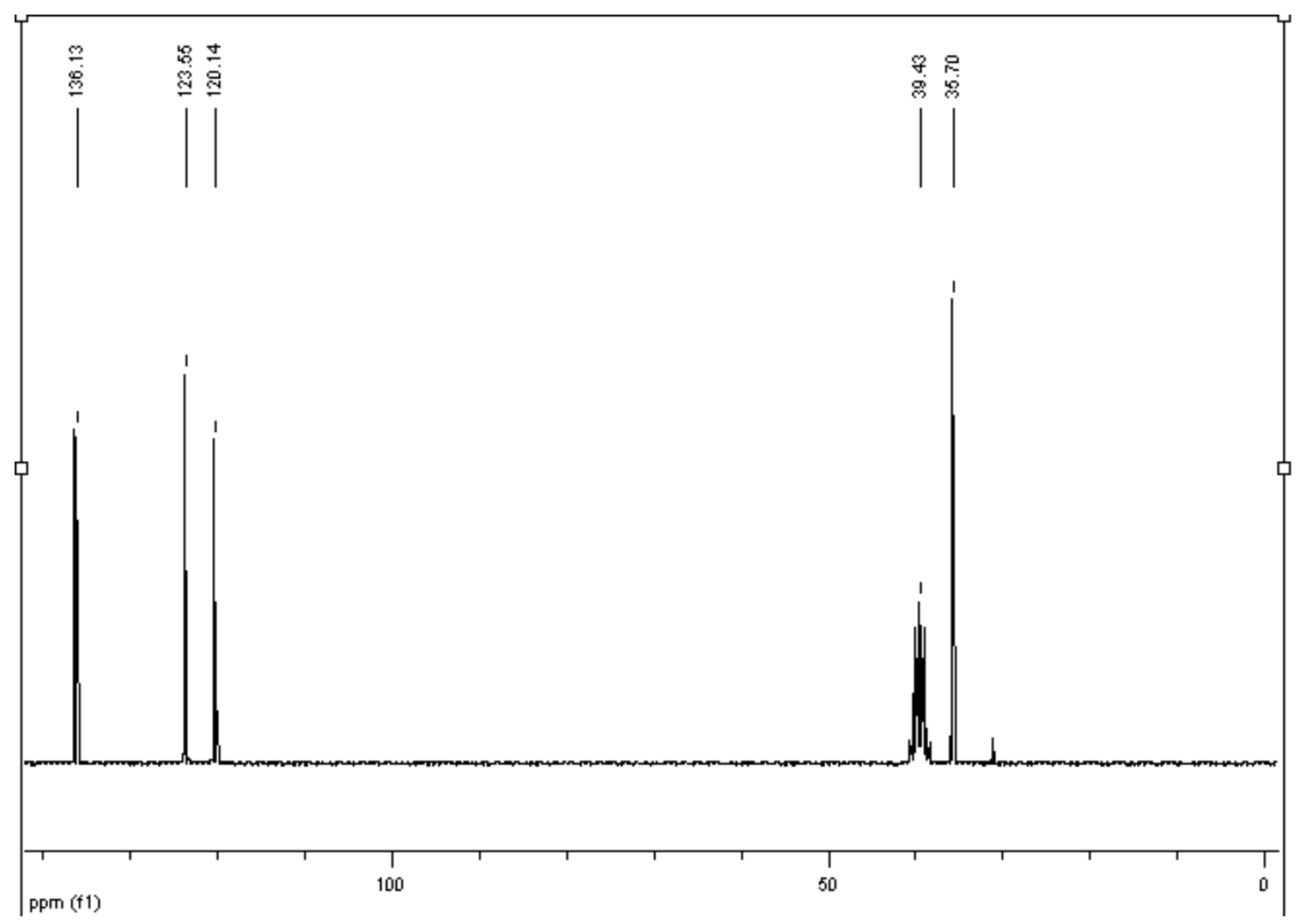


Figure S3. ${ }^{1} \mathrm{H}-\mathrm{NMR}$ spectrum of 1-butylimidazolium trifluoromethanesulfonate, $[\mathrm{Hbim}][\mathrm{TfO}]$ in dmso-d $_{6}(300 \mathrm{MHz})$.

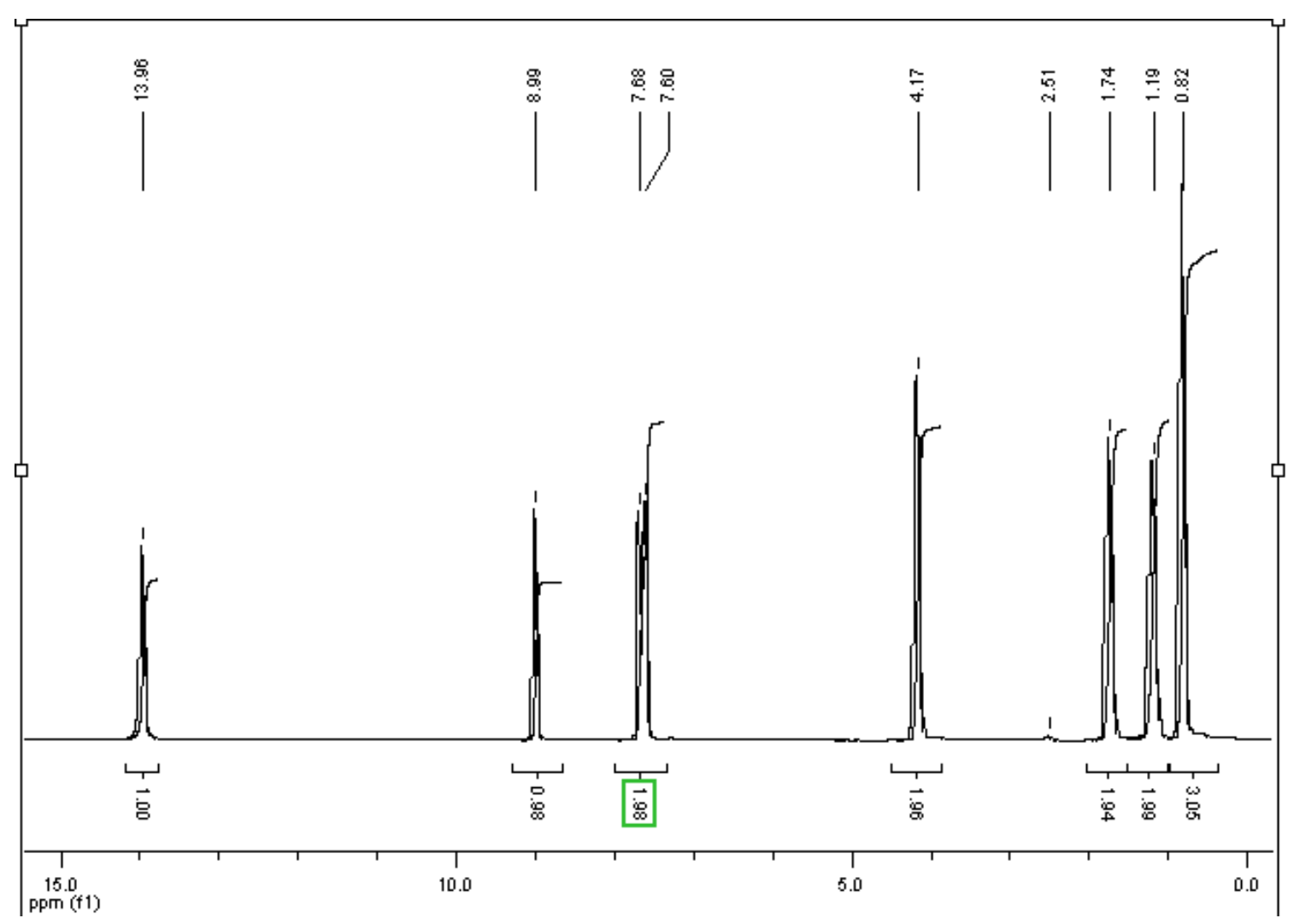


Figure S4: ${ }^{13} \mathrm{C}-\mathrm{NMR}$ spectrum of 1-butylimidazolium trifluoromethanesulfonate, [Hbim][TfO] in dmso-d $\mathrm{d}_{6}(75.47 \mathrm{MHz})$.

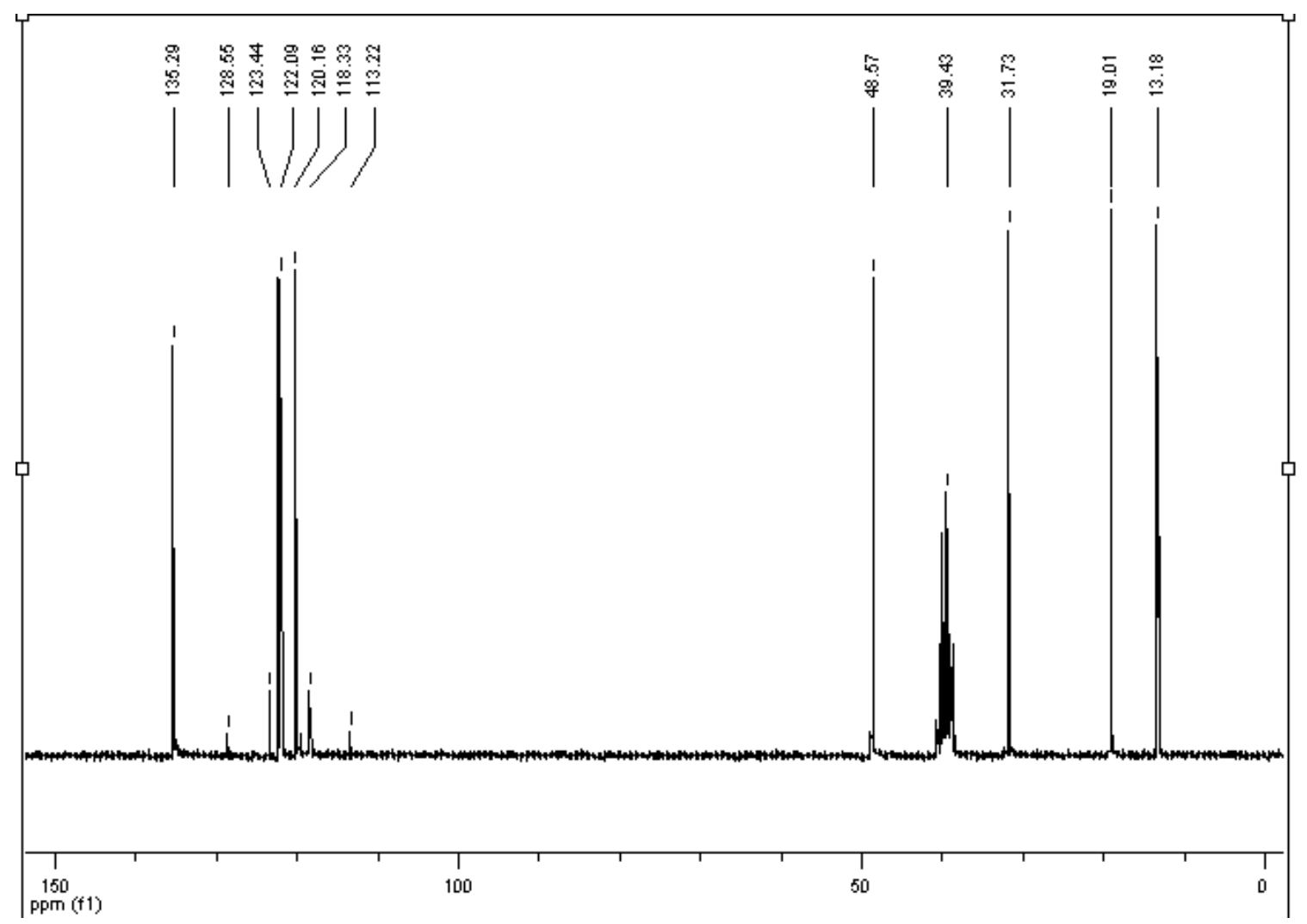


Figure S5. ${ }^{1} \mathrm{H}$-NMR spectrum of 1-hexyl-3-methylimidazolium trifluoromethanesulfonate, $\left[\mathrm{C}_{6} \mathrm{mim}\right][\mathrm{TfO}]$ in chloroform- $\mathrm{d}_{3}(300 \mathrm{MHz})$.

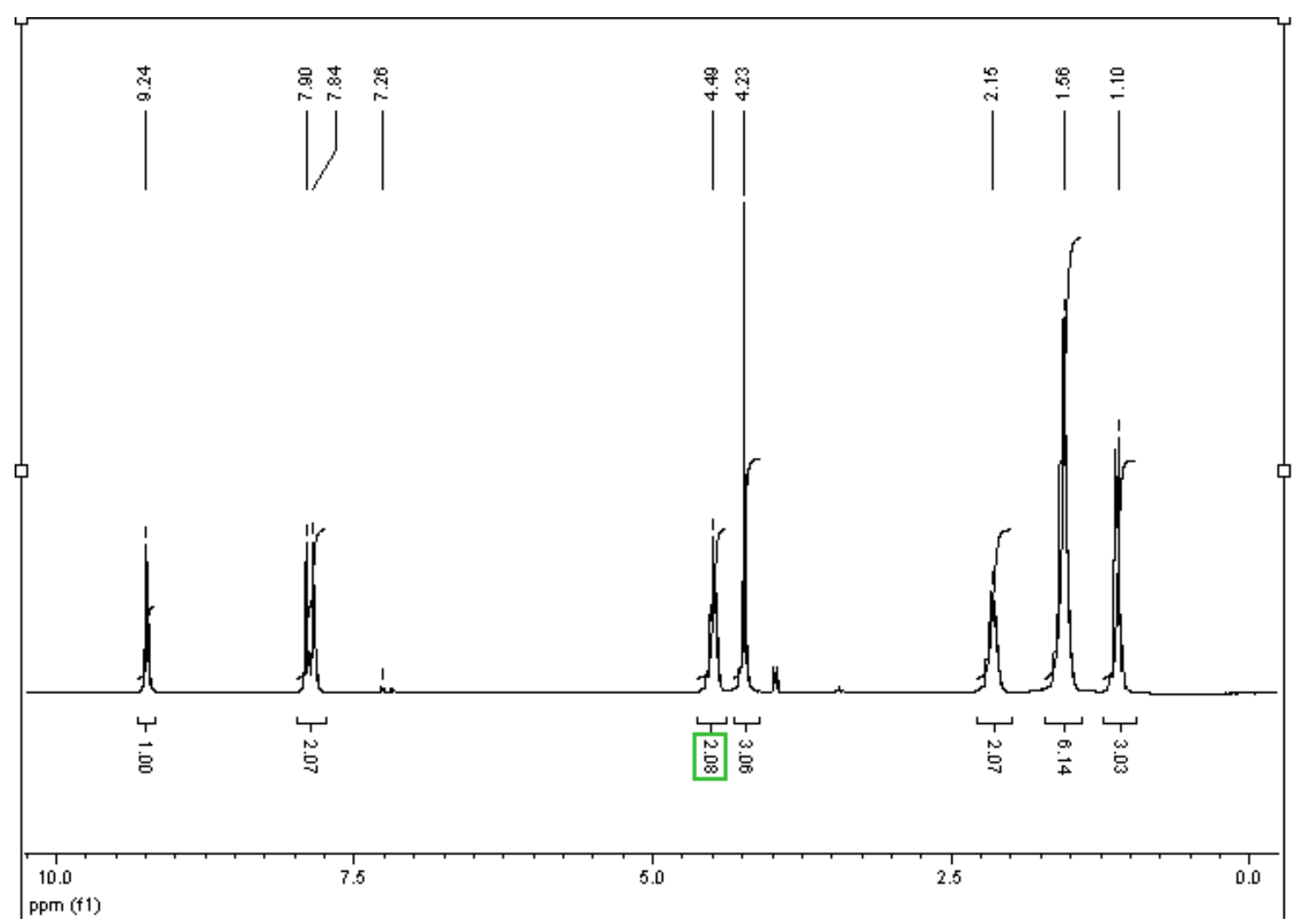


Figure S6. ${ }^{13} \mathrm{C}$-NMR spectrum of 1-hexyl-3-methylimidazolium trifluoromethanesulfonate, $\left[\mathrm{C}_{6} \mathrm{mim}\right][\mathrm{TfO}]$ chloroform- $\mathrm{d}_{3}(75.47 \mathrm{MHz})$.

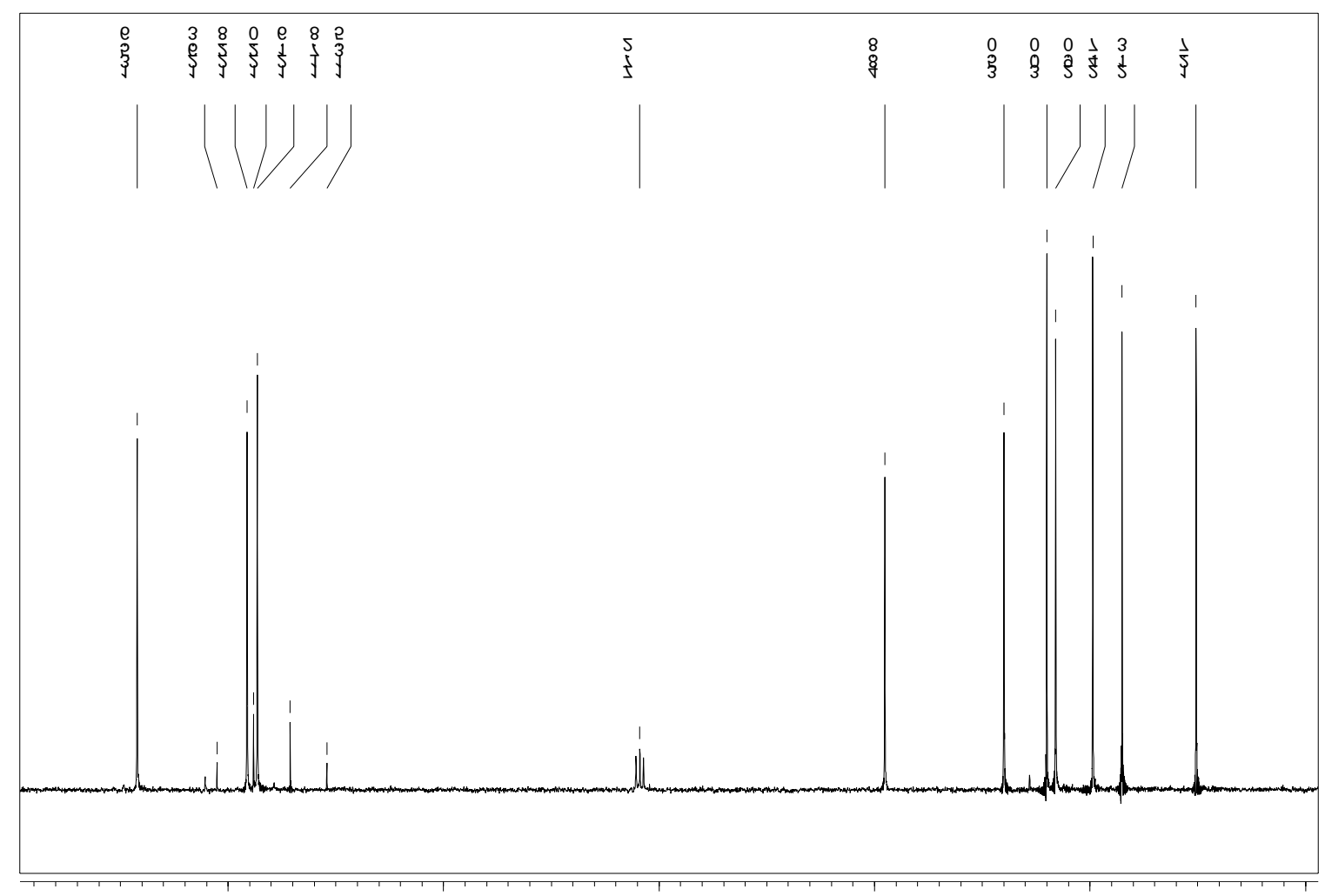


Figure S7. ${ }^{1} \mathrm{H}-\mathrm{NMR}$ spectrum of 1-methylimidazolium trifluoromethanesulfonate, [emim][TfO] in acetone- $\mathrm{d}_{6}(250 \mathrm{MHz})$.

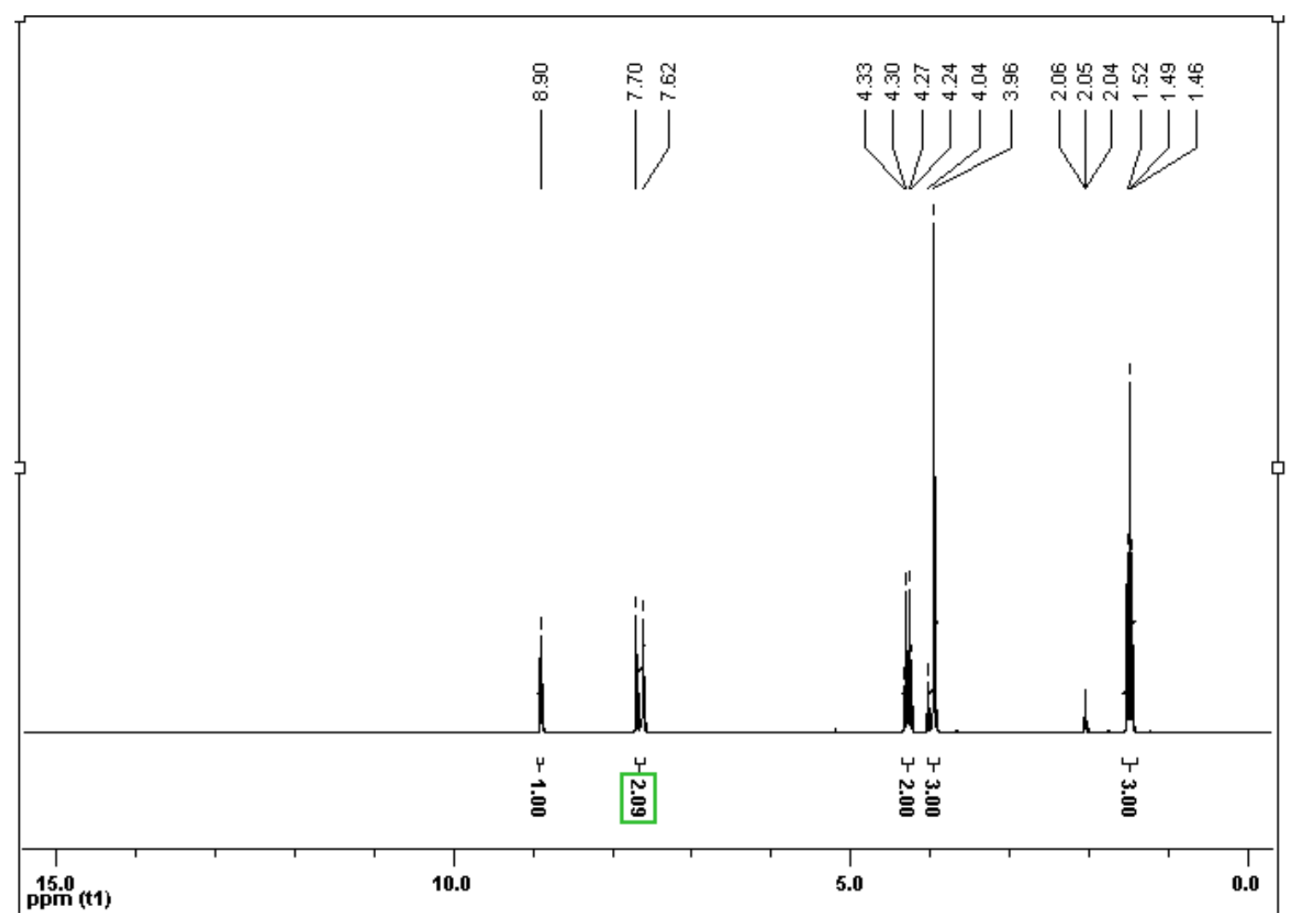


Figure S8. ${ }^{13} \mathrm{C}-\mathrm{NMR}$ spectrum of 1-methylimidazolium trifluoromethanesulfonate, [emim][TfO] in acetone- $\mathrm{d}_{6}(62.9 \mathrm{MHz})$.

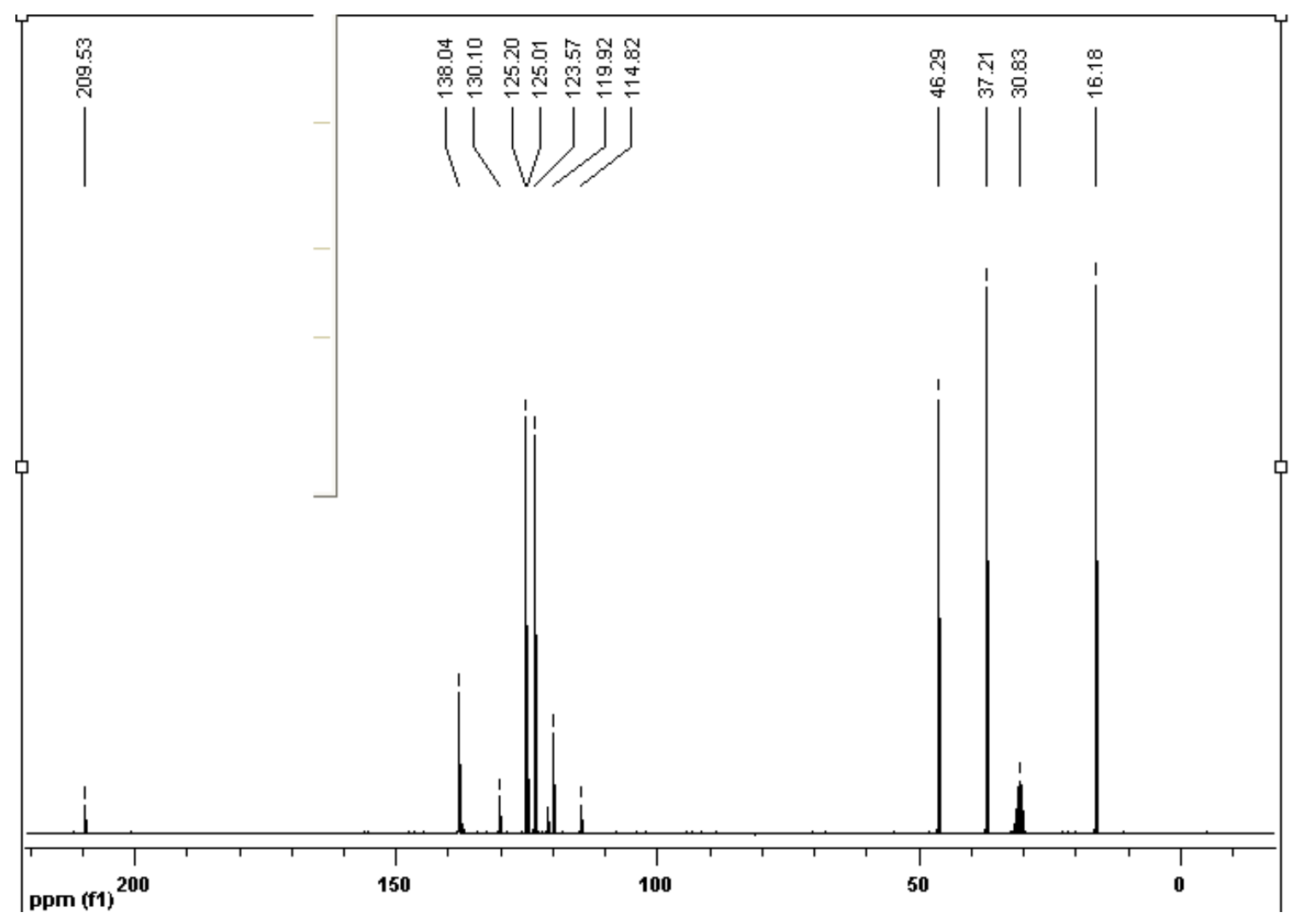


Figure S9. ${ }^{19}$ F-NMR spectrum of 1-methylimidazolium trifluoromethanesulfonate, [emim][TfO] in acetone- $\mathrm{d}_{6}(235.2 \mathrm{MHz})$.

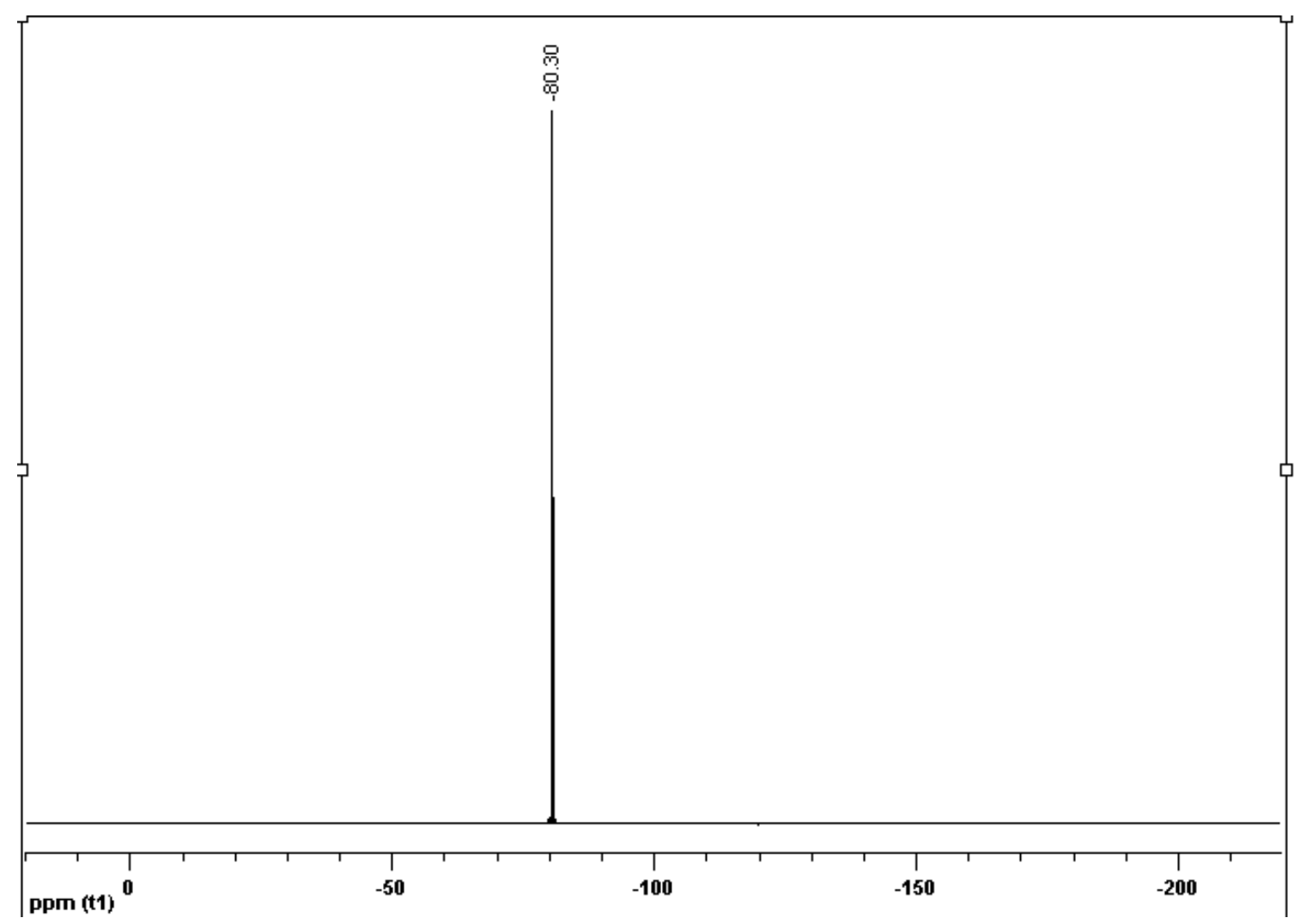

